\title{
Scheduling jobs with position-dependent processing times
}

\author{
A Janiak ${ }^{1}$ and MY Kovalyov ${ }^{2}$ \\ ${ }^{1}$ Wroclaw University of Technology, Wroclaw, Poland; and ${ }^{2}$ United Institute of Informatics Problems, \\ National Academy of Sciences of Belarus, Minsk, Belarus
}

Bachman and Janiak provided a sketch of the proof that the problem $1\left|r_{i}, p_{i}(v)=a_{i} / v\right| C_{\max }$ is NP-hard in the strong sense. However, they did not show how to avoid using harmonic numbers whose encoding is not pseudo-polynomial, which makes the proof incomplete. In this corrigendum, we provide a new complete proof.

Journal of the Operational Research Society (2012) 63, 1018-1020. doi:10.1057/jors.2012.18

Published online 25 April 2012

Keywords: sequencing; single machine; learning effect; computational complexity

Correction to: A Bachman and A Janiak (2004) Scheduling jobs with position-dependent processing times, Journal of the Operational Research Society 55: 257-264. doi:10.1057/palgrave.jors.2601689

In the problem $1\left|r_{i}, p_{i}(v)=a_{i} / v\right| C_{\max }$, there are $n$ jobs to be scheduled for processing on a single machine to minimize the makespan, $C_{\max }$. Each job $i$ is associated with a given release date $r_{i}$ before which its processing cannot start, and a position-dependent processing time $p_{i}(v)=a_{i} / v$, where $a_{i}$ is a given coefficient and $v$ is the position of job $i$ in the processing sequence. This dependence of processing times is called learning effect in the scheduling literature.

Our note is concerned with the proof of NP-hardness in the strong sense of the above problem. Similarly to Bachman and Janiak (2004), it is based on a reduction from the NP-hard in the strong sense problem 3-PARTITION, which can be defined as follows.

3-PARTITION: Given $3 m+1$ positive integer numbers $x_{1}, \ldots, x_{3 m}$ and $B$ such that $\sum_{j=1}^{3 m} x_{j}=m B$ and $B / 4<x_{j}<B / 2$ for $j=1, \ldots, 3 m$, is there a partition of the set $\{1, \ldots, 3 m\}$ into $m$ disjoint subsets $X_{l}$ such that $\Sigma_{j \in X_{l}} x_{j}=B$ for $l=1, \ldots, m$ ?

Dr Radoslaw Rudek pointed out to us that a sketch of the proof given in Bachman and Janiak (2004) is incorrect due to the usage of harmonic numbers $H_{l}=\sum_{k=1}^{l}(1) /(k)$, $l=1, \ldots, m$, in the calculation of the job release dates. Due to this, the release dates cannot be expressed as irreducible fractions of the type $A_{l} / B_{l}$ such that $A_{l}$ and $B_{l}$ are pseudopolynomially bounded in the length of 3-PARTITION, which means that Bachman and Janiak (2004) proves only NP-hardness in the ordinary sense of the problem $1\left|r_{i}, p_{i}(v)=a_{i} / v\right| C_{\max }$. We appreciate Dr Rudek's note. Below we demonstrate how to avoid the trouble with the harmonic numbers.
Theorem 1 The problem $1\left|r_{i}, p_{i}(v)=a_{i} / v\right| C_{\max }$ is NP-hard in the strong sense.

Proof We use the following reduction from 3-PARTITION. There are $3 m$ partition jobs and $m$ groups of enforcer jobs. For a partition job $i$ we have $r_{i}=0$ and $a_{i}=W^{2} x_{i}$, where $W$ is a sufficiently large number to be determined later. All the enforcer jobs have $a_{i}=1$. There are $g_{1}=$ $(W) /(m)$ enforcer jobs of Group 1 and $g_{l}=(W) /((m-l+1)$ $(m-l+2))$ enforcer jobs of Group $l, 2 \leqslant l \leqslant m$, where [ $\cdot]$ is the rounding up operator. Jobs of Group $l$ have the same release date $r_{l}$. There is a threshold $y$ on the $C_{\max }$ value. The values of the release dates and $y$ will be determined later such that $r_{1}=0$ and the interval $\left[r_{l}, r_{l+1}\right]$ can accommodate all the enforcer jobs of Group $l$ and some part of partition jobs for $l=1, \ldots, m$, where $r_{m+1}=y$. Note that the release date sequence is increasing.

Consider an arbitrary optimal schedule. Because of the learning effect, the total processing time of all jobs is minimized if the enforcer jobs (with small values $a_{i}$ ) are scheduled at the earliest possible times. As the enforcer jobs differ only by their release dates, they can always be interchanged to be sequenced in the non-decreasing order of their release dates. Therefore, assume without loss of generality that the groups of the enforcer jobs are scheduled in the order $1,2, \ldots, m$, and that jobs of Group $l$ are scheduled contiguously and start no earlier than at the time $r_{l}, l=1, \ldots, m$. Jobs of Group 1 start at time $r_{1}=0$.

Denote by $\delta_{l}$ the total processing time of the enforcer jobs of Group $l$ in the considered optimal 
schedule. Taking into account $g_{l} \leqslant W$ for $l=1, \ldots, m$, we obtain

$$
\begin{gathered}
\delta_{l} \leqslant H_{W}=\sum_{j=1}^{W} \frac{1}{j}<\ln W+\gamma+1<\ln W+2, \\
l=1, \ldots, m,
\end{gathered}
$$

where $H_{W}$ is a harmonic number and $\gamma$ is the EulerMascheroni constant. In contrast to Bachman and Janiak (2004), we will use an integer evaluation of the harmonic numbers rather than their exact values. Below we will show that this evaluation is sufficient for the proof.

Denote by $P_{l}$ the set of partition jobs scheduled between the enforcer jobs of Groups $l$ and $l+1$. Denote by $v_{i}$ the position of the partition job $i$. For $i \in P_{l}$ we have

$$
v_{i}=\sum_{j=1}^{l} g_{j}+\xi_{i},
$$

where $\xi_{i}$ is the number of partition jobs scheduled before job $i$ including this job. By using the definitions of the $g_{j}$ values and evaluating the rounding up operators, we obtain

$$
\begin{aligned}
v_{i} & =\frac{W}{m}+\sum_{j=2}^{l} \frac{W}{(m-j+1)(m-j+2)}+\chi_{i}+\xi_{i} \\
& =\frac{W}{m-l+1}+\psi_{i},
\end{aligned}
$$

where $0 \leqslant \chi_{i} \leqslant l$ is the value coming from discarding the rounding up operators, $\psi_{i}=\chi_{i}+\xi_{i}$, and $\sum_{j=2}^{l}(W)$ $((m-j+1)(m-j+2)):=0$ if $l=1$. It is easy to notice that $1 \leqslant \xi_{i} \leqslant \psi_{i} \leqslant 3 m+l \leqslant 4 m$, as $1 \leqslant \xi_{i} \leqslant 3 m$.

Calculate the total processing time of the partition jobs of the set $P_{l}$ and enforcer jobs of Group $l$, denoted as $T_{l}$.

$$
\begin{aligned}
T_{l}= & \sum_{i \in P_{l}} p_{i}\left(v_{i}\right)+\delta_{l}=\sum_{i \in P_{l}} \frac{a_{i}}{v_{i}}+\delta_{l} \\
= & \sum_{i \in P_{l}} \frac{W^{2} x_{i}}{\frac{W}{m-l+1}+\psi_{i}}+\delta_{l} \\
= & W(m-l+1) \sum_{i \in P_{l}} x_{i}+\delta_{l}-(m-l+1)^{2} \\
& \times \sum_{i \in P_{l}} \frac{\psi_{i} x_{i}}{1+\frac{\psi_{i}(m-l+1)}{W}}, \quad l=1, \ldots, m .
\end{aligned}
$$

The transition between the two lines in Equation (1) is justified by the following chain of equalities.

$$
W(m-l+1)-\frac{(m-l+1)^{2} \psi_{i}}{1+\frac{\psi_{i}(m-l+1)}{W}}
$$

$$
\begin{aligned}
& =\frac{W(m-l+1)\left[W+(m-l+1) \psi_{i}\right]-(m-l+1)^{2} \psi_{i} W}{W+\psi_{i}(m-l+1)} \\
& =\frac{(m-l+1) W^{2}}{W+\psi_{i}(m-l+1)}=\frac{W^{2}}{\frac{W}{m-l+1}+\psi_{i}} .
\end{aligned}
$$

Observe that for any schedule feasible with respect to the release dates, $C_{\max } \leqslant y$ if and only if

$$
\sum_{j=l}^{m} T_{j} \leqslant y-r_{l}, \quad l=m, m-1, \ldots, 1 .
$$

We choose $r_{1}, \ldots, r_{m}$ and $y=r_{m+1}$ such that $r_{1}=0$ and $r_{l}-r_{l-1}=B+W B(m-l+2), l=2, \ldots, m+1$. Hence, $y=m B+W B(m+1) m / 2$ and $y-r_{l}=(m-l+1) B+W B$ $\sum_{j=1}^{m-l+1} j, l=1, \ldots, m$.

By substituting Equation (1) into Equation (2), we obtain that for the considered optimal schedule $C_{\max } \leqslant y$ if and only if

$$
\begin{aligned}
\sum_{j=l}^{m} T_{j}= & W \sum_{j=l}^{m}\left((m-j+1) \sum_{i \in P_{j}} x_{i}\right) \\
& +\sum_{j=l}^{m}\left(\delta_{j}-(m-j+1)^{2} \sum_{i \in P_{j}} \frac{\psi_{i} x_{i}}{1+\frac{\psi_{i}(m-j+1)}{W}}\right) \\
\leqslant & y-r_{l}=(m-l+1) B+W B \sum_{j=1}^{m-l+1} j, \\
& l=m, m-1, \ldots, 1 .
\end{aligned}
$$

Rearrange Equation (3) so that

$$
\begin{gathered}
\sum_{j=l}^{m}\left((m-j+1) \sum_{i \in P_{j}} x_{i}\right) \leqslant B \sum_{j=1}^{m-l+1} j+D_{l}, \\
l=m, m-1, \ldots, 1,
\end{gathered}
$$

where

$$
D_{l}=\frac{(m-l+1) B+\sum_{j=l}^{m}\left((m-j+1)^{2} \sum_{i \in P_{j}} \frac{\psi_{i} x_{i}}{1+\frac{\psi_{i}(m-j-1)}{W}}-\delta_{j}\right)}{W},
$$

We would like to have $0 \leqslant D_{l}<1, l=1, \ldots, m$. Consider the following chain of relations:

$$
\begin{aligned}
& (m-j+1)^{2} \sum_{i \in P_{j}} \frac{\psi_{i} x_{i}}{1+\frac{\psi_{i}(m-j+1)}{W}} \\
& \leqslant m^{2} \sum_{i \in P_{j}}\left(\psi_{i} x_{i}\right) \leqslant 4 m^{3} \sum_{i \in P_{j}} x_{i} \leqslant 12 m^{4} B .
\end{aligned}
$$


Then $D_{l} \leqslant\left((m-l+1) B-\sum_{j=l}^{m} \delta_{j}+12 m^{5} B\right) /(W)$. We set $W=12 m^{5} B+m B+1$ and assume without loss of generality that $\ln W+2 \leqslant B$, which implies $\delta_{j} \leqslant B$ for all $j$. Here we use the integer evaluation of the harmonic numbers. Then $0 \leqslant(m-l+1) B-\sum_{j=l}^{m} \delta_{j} \leqslant m B$ and $0 \leqslant D_{l}<1, l=1, \ldots, m$.

Due to the integrality of the left-hand side and the first summand of the right-hand side in Equation (4), relations (4) are equivalent to

$$
\sum_{j=1}^{m-l+1} j \sum_{i \in P_{m-j+1}} x_{i} \leqslant B \sum_{j=1}^{m-l+1} j, \quad l=m, m-1, \ldots, 1
$$

By making index substitution $h:=m-l+1$, the latter relations can be expressed as:

$$
\sum_{j=1}^{h} j\left(B-\sum_{i \in P_{m-j+1}} x_{i}\right) \geqslant 0, \quad h=1, \ldots, m .
$$

Suppose that at least one of the inequalities in Equation (5) is strict. Then by forming a linear combination of the $m$ inequalities with positive coefficients $1 / h-1 /$ $(h+1)$ for $h=1, \ldots, m-1$, and $1 / m$ for $h=m$, we obtain a strictly positive value

$$
\begin{gathered}
\sum_{h=1}^{m-1}\left(\frac{1}{h}-\frac{1}{h+1}\right) \sum_{j=1}^{h} j\left(B-\sum_{i \in P_{m-j+1}} x_{i}\right) \\
+\frac{1}{m} \sum_{j=1}^{m} j\left(B-\sum_{i \in P_{m-j+1}} x_{i}\right)>0 .
\end{gathered}
$$

The latter expression can be rearranged as follows:

$$
\begin{aligned}
& \sum_{j=1}^{m} j\left(B-\sum_{i \in P_{m-j+1}} x_{i}\right)\left(\sum_{h=j}^{m-1}\left(\frac{1}{h}-\frac{1}{h+1}\right)+\frac{1}{m}\right) \\
& =m B-\sum_{j=1}^{m} \sum_{i \in P_{m-j+1}} x_{i}>0,
\end{aligned}
$$

which is a contradiction because $\sum_{j=1}^{m} \sum_{i \in P_{m-j+1}} x_{i}=m B$ by the definition of 3-PARTITION. Hence, the left-hand side of each inequality in Equation (5) is equal to 0, which implies that 3-PARTITION has a solution if and only if for the considered optimal schedule $C_{\max } \leqslant y$.

An evident observation that our reduction is pseudopolynomial in the length of 3-PARTITION completes the proof.

\section{Reference}

Bachman A and Janiak A (2004). Scheduling jobs with positiondependent processing times. Journal of the Operational Research Society 55(3): 257-264. 